\title{
Characteristics of the flow over a NACA 0012 airfoil at low Reynolds numbers
}

\author{
R. W. Derksen, M. Agelinchaab \& M. Tachie \\ Department of Mechanical and Manufacturing Engineering, University \\ of Manitoba, Winnipeg, Manitoba, Canada
}

\begin{abstract}
This paper presents our work of an experimental examination of the flow over a NACA 0012 Airfoil at low Reynolds numbers and large angle of attack using particle imaging velocimetry. The Reynolds numbers examined were 5,000, 30,000 , and 60,000 , while the angles of attack ranged from 8 to 12 degrees in 2 degree increments. This work was motivated by reports that lift and drag measurements for airfoils operating at Reynolds numbers less than roughly 40,000 could not be made due to flow unsteadiness. This is puzzling in that the flow should be laminar at these Reynolds numbers, which are an order of magnitude lower than the flat-plate transition Reynolds number of 500,000. To this end we examined a sequence of flow field measurements of the instantaneous velocity field. We observed mean streamline patterns that were very representative of those we would find for a strictly steady flow, however a random pattern of significant fluctuations in the velocity and vorticity were observed. The intensity of these fluctuations increased with Reynolds number and angle of attack.
\end{abstract}

Keywords: aerodynamics, low Reynolds number flow, particle imaging velocimetry.

\section{Introduction}

Historically, interest in low Reynolds number aerodynamics has been restricted to model radio controlled aircraft applications and did not garner a great deal of scientific interest. This situation is changing with the interest in small unmanned aerial vehicles, predominantly for military purposes. As such, our understanding of low Reynolds number flow over airfoils is limited to a few sources that are 
growing as time evolves. This naturally leads us to the outstanding work done by Selig et al [1-4] in the mid-nineties. This work details the results of a carefully constructed experimental program to produce a database of modern, low-Reynolds number airfoils. The measurements were exhaustively examined for their accuracy, for example drag was measured by the more accurate wake traverse method.

One observation made in the Selig work was that they could not reliably measure airfoil characteristics if the Reynolds number fell below 40,000 due to flow unsteadiness. Huang and Lin [5] clearly demonstrated vortex shedding and instability at low-Reynolds numbers using smoke-streak and hot-wire measurements of the flow over a NACA 0012 airfoil. Unfortunately, the smokestreak method presents us with a time averaged view of the flow field and hotwire only yields point observations. We suspected that this may be the result of a periodic bursting of a laminar separation bubble. In this process the separation bubble would develop to such a point that it would detach from the airfoil and move downstream. Once the remnant of the bubble was sufficiently far downstream the process would then repeat periodically. A computational study of unsteady boundary-layer separation over an Eppler 387 airfoil at lowReynolds numbers done by Lin and Pauley [6] appears to support this view.

We felt that this was a sufficiently interesting possible phenomenon that deserved an in depth study which is described below. The main objective of this study was to determine the characteristics of the bubble growth, that triggered the detachment, to observe the movement of the detached bubble.

\section{Experimental set-up and procedure}

The experiments were based on the flow over a NACA 0012 symmetric airfoil, which is the same as that studied by Huang and Lin. This airfoil is often used in aerodynamic studies and forms a good base to work from.

\subsection{Test section}

The experiments were performed in a re-circulating open channel with a test section that is $2500 \mathrm{~mm}$ long, $200 \mathrm{~mm}$ wide and $200 \mathrm{~mm}$ deep. A provisional closed test section $2500 \mathrm{~mm}$ long with a $187 \mathrm{~mm} \times 187 \mathrm{~mm}$ cross-section was inserted into the open channel to hold the airfoil in place. The airfoil is symmetric and has a chord length of $100 \mathrm{~mm}$, a maximum thickness of $12 \mathrm{~mm}$ and a width of $187 \mathrm{~mm}$ that spans the entire width of the test section. The airfoil was tightly screwed to the side walls of the test section at $1000 \mathrm{~mm}$ downstream from the inlet of the channel to ensure uniform flow at the measurement section. Figure 1 is the schematic of the provisional closed test section with the laser and camera arrangement. 


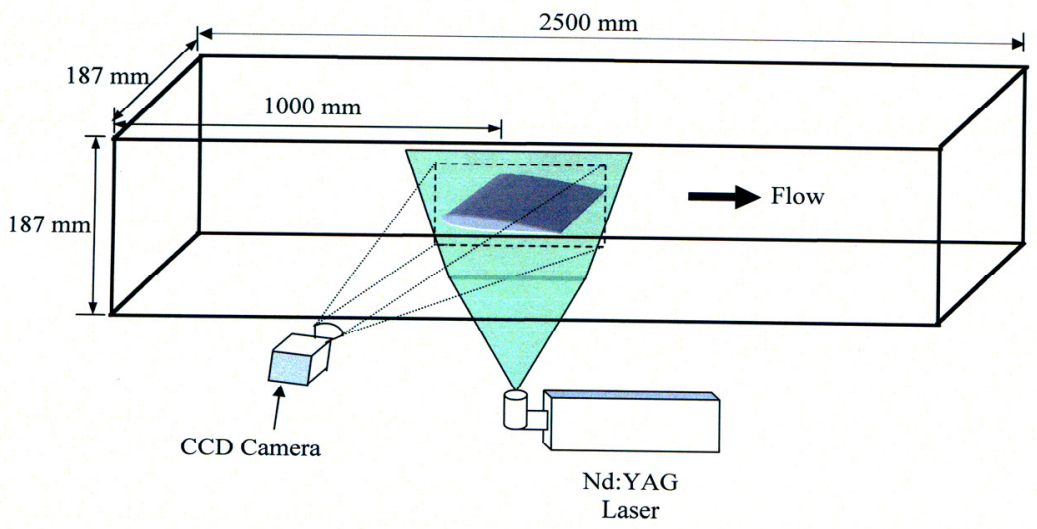

Figure 1: $\quad$ Experimental set-up of the test section, airfoil, CCD camera and laser arrangement.

\subsection{Measurement procedure}

A particle image velocimetry (PIV) technique was used for the velocity measurements. The flow was seeded with $5 \mu \mathrm{m}$ polyamide seeding particles. An $\mathrm{Nd}-\mathrm{YAG}, 120 \mathrm{~mJ} /$ pulse laser of $532 \mathrm{~nm}$ wavelength was used to illuminate the flow field. A 12-bit high-resolution CCD camera with 2048 pixels $\times 2048$ pixels and a $7.4 \mu \mathrm{m}$ pixel pitch was employed to image the flow field. The instantaneous images were processed using the adaptive correlation option of FlowManager (version 4.50.17) developed by Dantec Dynamics. A 32 pixels $\times$ 16 pixels interrogation window (IW) with $50 \%$ overlap and moving average validation was used. The adaptive correlation used a multi-pass FFT crosscorrelation algorithm to determine the average particle displacement within the IW. A three-point Gaussian curve fit was employed to determine particle displacement with sub-pixel accuracy. The particle image diameter was estimated to be $d_{p}=2.1$ pixels, a value that is very close to the recommended optimum value of $d_{p} \approx 2$ pixels required to minimize peak locking (Raffel et al [7]).

Whereas the vorticity contours are instantaneous, the mean streamlines, turbulent intensities, and Reynolds shear stress were obtained from a sample size of 250 images. A field of view was made large enough to capture the whole airfoil at once. The physical spacing between data points along the flow direction and normal to the flow direction were $1.2 \mathrm{~mm}$ and $0.6 \mathrm{~mm}$, respectively.

The Reynolds numbers based on the chord length and the upstream velocities were $R e=5000,30,000$ and 60,000. For each $R e$, the angle of attack was varied from $\theta=8^{\circ}, 10^{\circ}$ and $12^{\circ}$ to investigate the effect of both $R e$ and $\theta$ on the airfoil. The measurements were obtained at the mid-plane of the test section. 


\subsection{Measurement uncertainty}

Uncertainty analysis was made following the AIAA standard derived and explained by Coleman and Steele [8]. In general, a complete uncertainty analysis involves identifying and quantifying both the bias and precision errors in each part of the measurement chain. In a PIV technique, the accuracy of velocity measurement is limited by the accuracy of the sub-pixel interpolation of the displacement correlation peak. Other sources of measurement uncertainties include particle response to fluid motion, light sheet positioning, light pulse timing and size of interrogation area. Detailed analyses of bias and precision errors inherent in the PIV technique are available in Prasad [9] and Forliti et al [10]. Forliti et al showed that a Gaussian peak-fitting algorithm has the lowest bias and precision errors. On the basis of the size of the interrogation area and the curve fitting algorithm used to calculate the instantaneous vector maps, the uncertainty in the mean velocities at a $95 \%$ confidence level was estimated to be $\pm 2 \%$, in turbulence intensities are estimated to be $\pm 7 \%$, and in Reynolds shear stress are estimated to be $\pm 10 \%$.

\section{Results}

A survey of the results will be given due to the volume of data that was produced and the limited space available in this document. The characteristics of the flow can be summarized as follows. The mean stream lines were as to be expected for a steady laminar flow for each angle of attack and for each of the three Reynolds numbers. The flow was attached to the airfoil for $\theta=8$ and $10^{\circ}$, but a small separation bubble was evident for the $12^{\circ}$ case. This bubble was most noticeable for $\mathrm{Re}=5,000$ and diminished as the Reynolds number increased to the point that it was barely detectible for $\mathrm{Re}=60,000$. Generally, the intensity of the disturbances, as indicated by the turbulence intensity, increased with Reynolds number for a fixed angle of attack. However there was a pronounced increase in both intensity and amount of the flow field affected by the disturbance for $\mathrm{Re}=60,000$. A snap shot of the instantaneous vorticity field corresponds to this picture. It is interesting to note that the region that contains fluctuating vorticity is usually smaller and more dispersed than that indicated by the mean turbulence intensity field, indicating that the mean intensities indicate a greater influence of the disturbances that occurs at any given time. One would be tempted to think that this would indicate the presence of turbulence in the flow. However, with the exception of the $\operatorname{Re}=60,000$ and $\theta=12^{\circ}$ case, the mean Reynolds shear stresses were very small - virtually negligible. This would tend to indicate that an instability mechanism is at play generating random fluctuations, but that the flow has not developed a correlated turbulence region. We will focus on the $\mathrm{Re}=5,000$ cases for our detailed discussion that follows.

\subsection{Mean streamlines at $\operatorname{Re}=5,000$}

The mean streamlines for $\mathrm{Re}=5,000$ are shown below, in Figure 2 . 


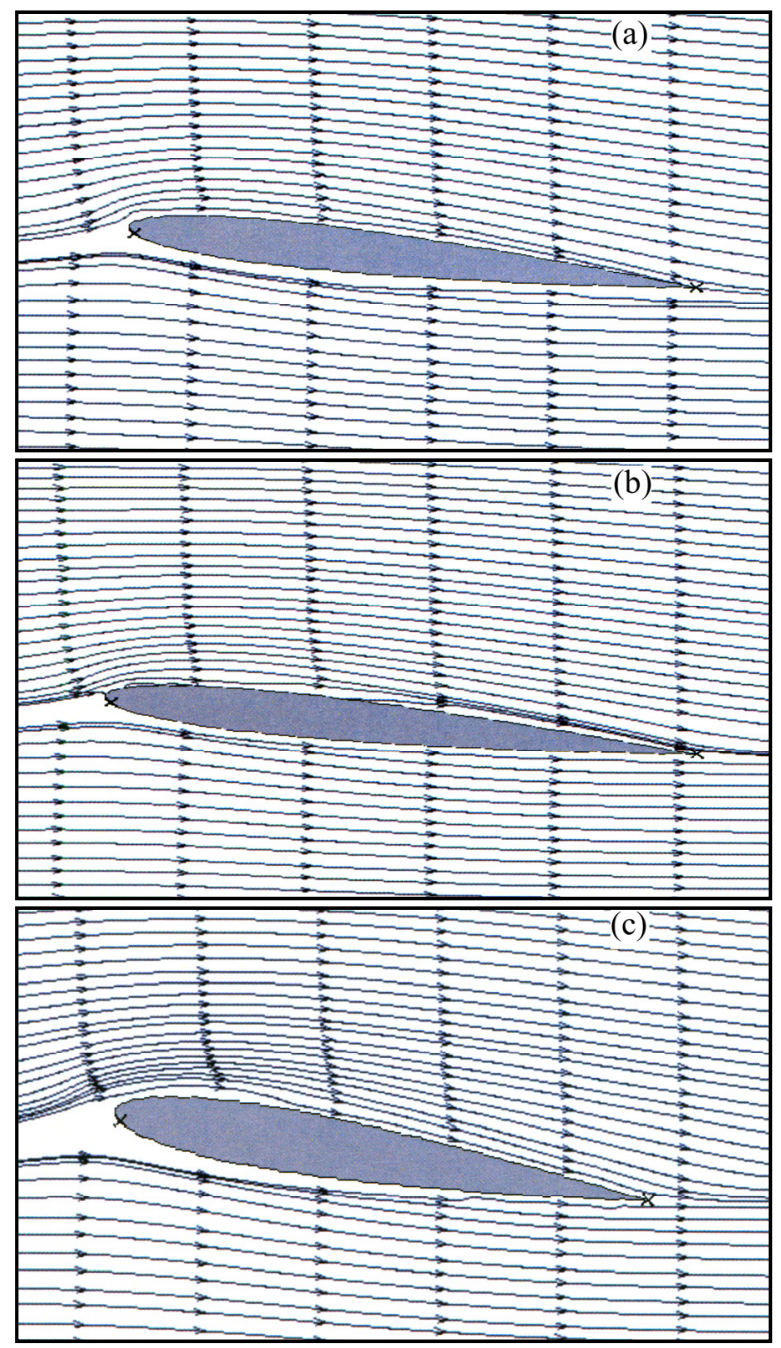

Figure 2: $\quad$ The mean streamlines at $\operatorname{Re}=5,000$; (a) $\theta=8^{\circ}$, (b) $\theta=10^{\circ}$, and (c) $\theta=12^{\circ}$.

This figure clearly demonstrates the characteristic streamline pattern that we would expect to see for a steady laminar flow. The flow is generally attached, but a separation bubble appears at $\theta=12^{\circ}$ which corresponds to the known maximum lift angle of attack. This picture is consistent with the results for $\mathrm{Re}=30,000$ and 60,000. 


\subsection{Turbulence intensities at $\mathrm{Re}=\mathbf{5 , 0 0 0}$}

The measured turbulence intensities are shown below in Figure 3 for $\mathrm{Re}=5,000$.

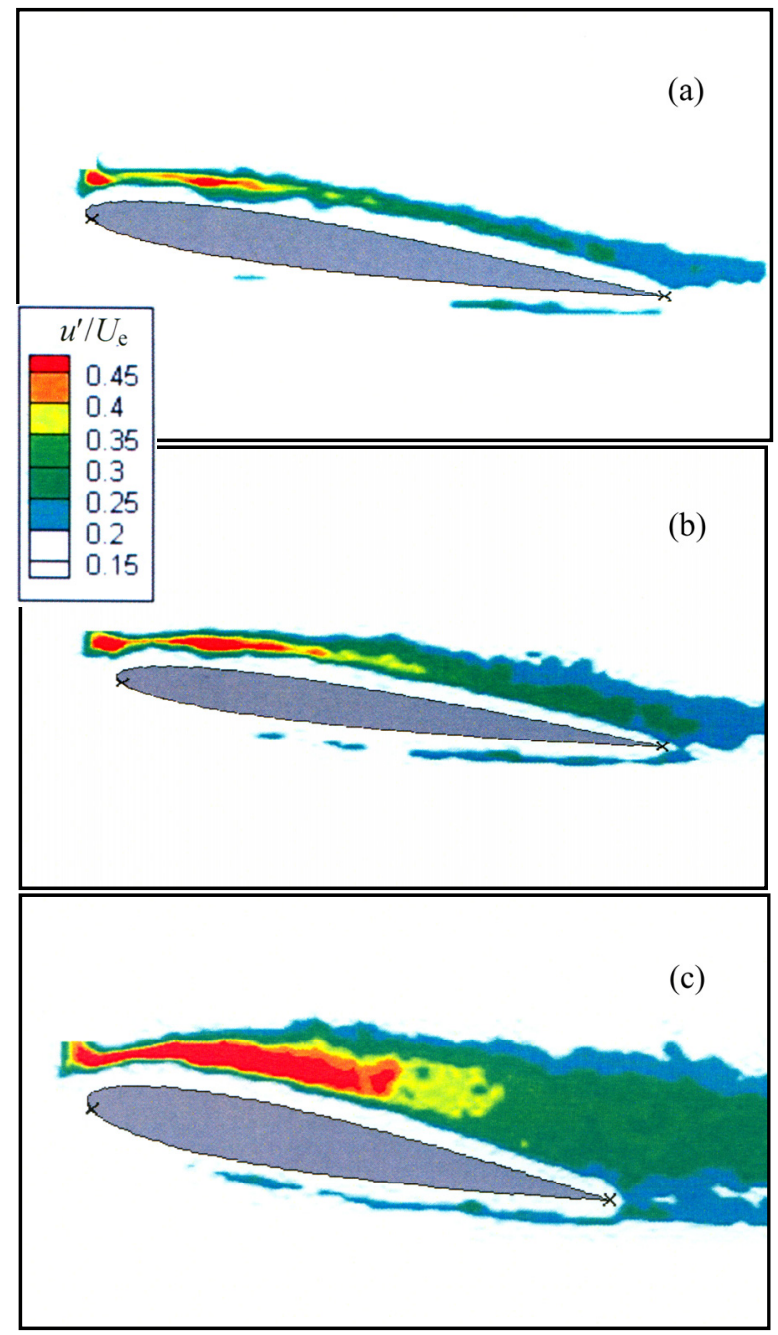

Figure 3: $\quad$ The turbulence intensities at $\operatorname{Re}=5,000$; (a) $\theta=8^{\circ}$, (b) $\theta=10^{\circ}$, and (c) $\theta=12^{\circ}$.

Figure 3 clearly demonstrates a zone of fluctuation that is most noticeable above the suction surface and in particular above the leading portions of the airfoil. This zone is physically separated from the surface of the airfoil at a distance approximately equal to its width. At the largest Reynolds number, 60,000, it appears that the affected zone grows dramatically in intensity and size. 


\subsection{Instantaneous vorticity distributions at $\operatorname{Re}=\mathbf{5 , 0 0 0}$}

Instantaneous snapshots of the vorticity for $\mathrm{Re}=5,000$ are shown below in Figure 4.

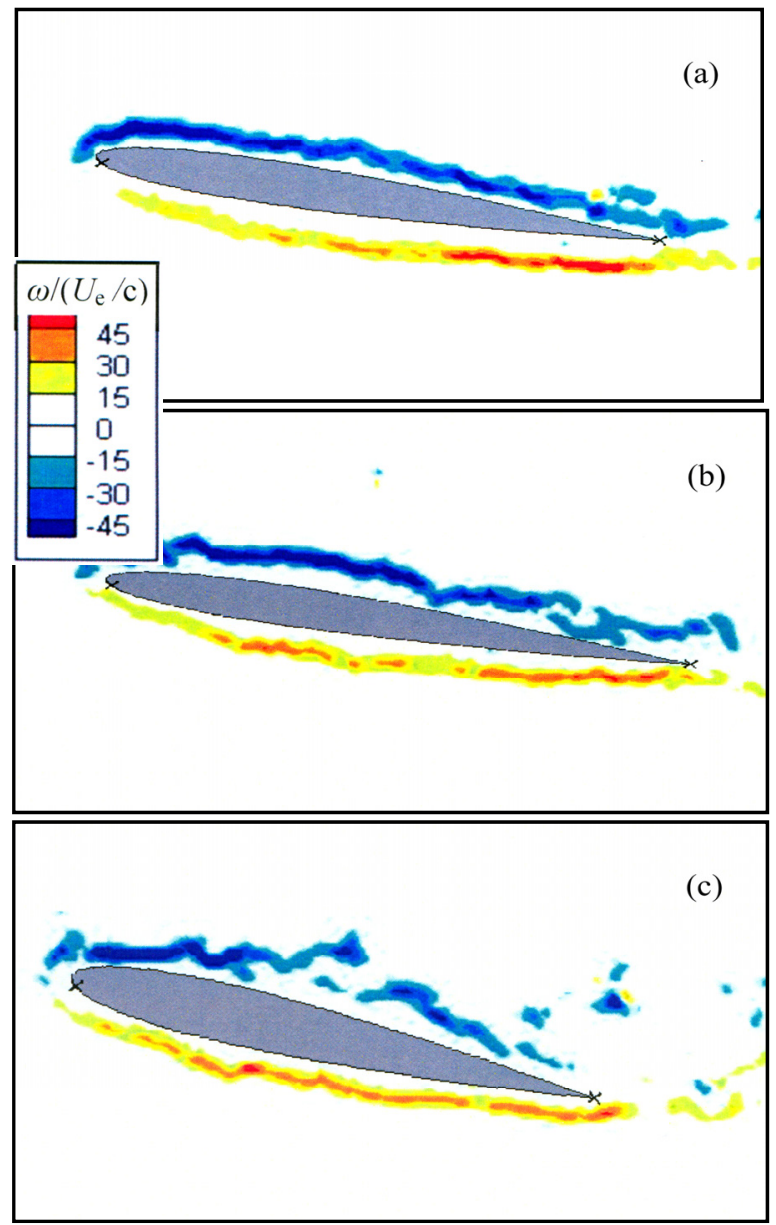

Figure 4: $\quad$ A snap shot of the instantaneous vorticity at $\operatorname{Re}=5,000$; (a) $\theta$ $=8^{\circ}$, (b) $\theta=10^{\circ}$, and (c) $\theta=12^{\circ}$.

Figure 4 clearly demonstrates the limited size of the zone affected by the disturbances at any given time. In all cases the size of the region is similar; however, the meander of the instantaneous vorticity clearly increases with Reynolds number. Notice that the size of the region of the flow that is affected by the disturbances is significantly smaller than that indicated by the size of the regions with notable turbulence intensity. 


\subsection{Reynolds shear stress at $\mathrm{Re}=\mathbf{5 , 0 0 0}$}

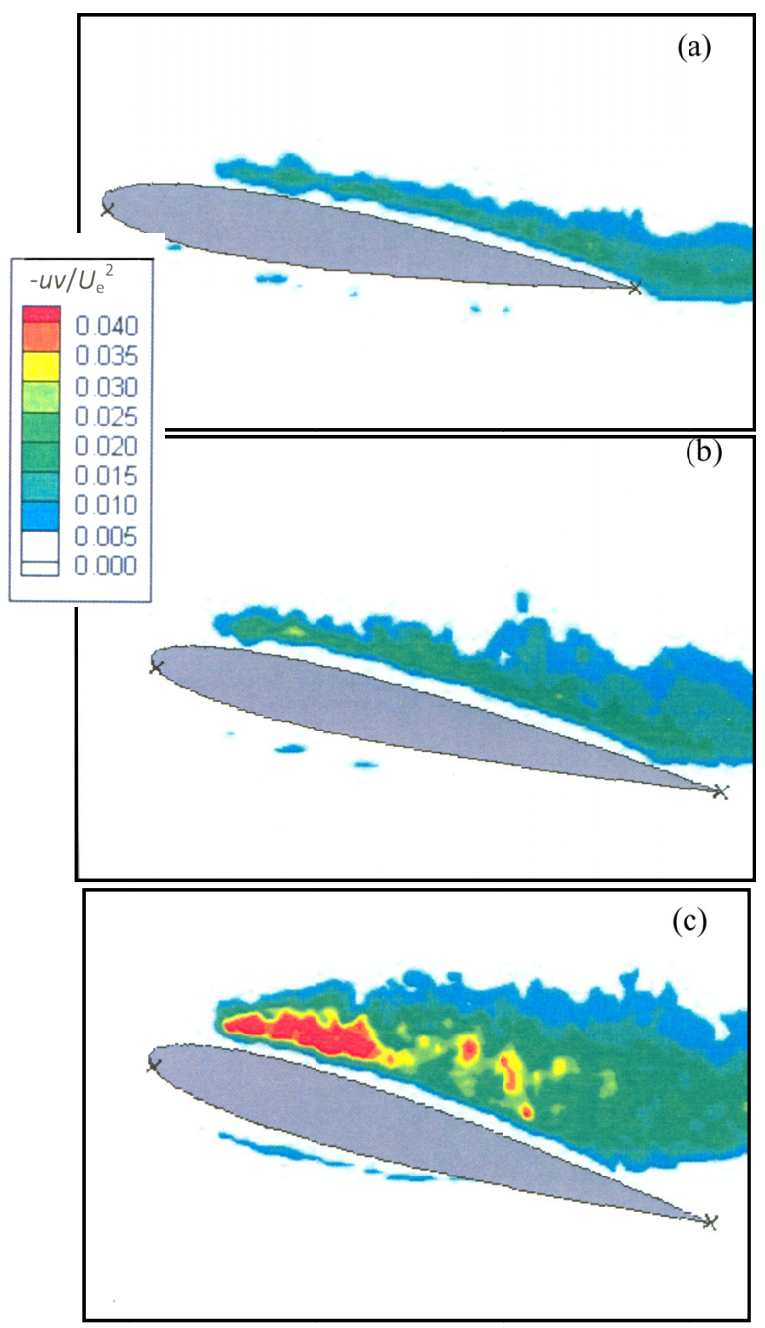

Figure 5:

The Reynolds shear stresses at $\operatorname{Re}=5,000$; (a) $\theta=8^{\circ}$, (b) $\theta=10^{\circ}$, and (c) $\theta=12^{\circ}$.

Figure 5 is a flow field map of the mean Reynolds stresses for $\mathrm{Re}=5,000$. Here we clearly see that the turbulence levels are negligible for the lower angles of attack, however at the higher angle of attack a significant degree of Reynolds stress is evident above the forward portion of the suction surface. 


\section{Discussion}

An examination of the mean streamline patterns about the airfoils does not indicate any of the rich character of the flow. They are very similar to what we would expect if the flow was a strictly steady, laminar flow, as we would expect based on the low Reynolds numbers studies.

The results of this study clearly demonstrate that significant levels of fluctuations exist in the flow over a NACA 0012 airfoil at angles of attack between 8 and $12^{\circ}$. This is true even though the Reynolds numbers were very small, well below the typically turbulent transition Reynolds number of 500,000. Clearly, an instability mechanism is at play and we cannot assume that the flow is automatically steady. The magnitudes of the disturbances are too large to consider as minor background disturbances, which are not observed in the main body of the flow away from the airfoil.

The snapshots of the instantaneous vorticity distributions clearly indicate that similar zones are affected by the disturbances as indicated by the mean turbulence intensities in all cases. For $\mathrm{Re}=5,000$ and 30,000 the instantaneously affected zones are very similar to that indicated by the mean turbulence intensity. However, at the highest Reynolds number the instantaneous vorticity affected zone meanders about the flow field considerably, causing the affected zone to appear much larger when we examine the mean turbulence intensities. The highest Reynolds number and angle of attack could result in transition as this angle of attack approximately corresponds to the maximum lift point.

The measured Reynolds stresses confirm the picture that the flow is not turbulent, at least for the lower angles of attack and Reynolds numbers. In general, we observe very low degrees of correlation between the stream wise and normal components of the velocity fluctuations. This is more indicative of a random, uncorrelated disturbance pattern that likely is the result of a flow instability mechanism. It also indicates that the mean streamline field would not be affected by these disturbances, as was observed.

The picture does appear to change somewhat at the highest angle of attack of $12^{\circ}$, particularly at $\mathrm{Re}=60,000$. This may indicate that the transition Reynolds number for this airfoil at this angle of attack is about 60,000 .

\section{Conclusions}

The results of this work present us with three interesting and somewhat unexpected observations.

First there is clear evidence of significant levels of flow disturbances in all cases examined, with some indication that there may be some turbulent flow at $\mathrm{Re}=60,000$ and $\theta=12^{\circ}$. The consequences of this is that while our basic flow model of steady laminar flow will result in reasonably good predictions of mean behaviour, the flow is still highly unsteady, complicating measurements of the airfoil's characteristics. 
The second observation is that the flow is not turbulent, except for the $12^{\circ}$, $\mathrm{Re}=60,000$ case. The correlation between the stream-wise and normal velocity components is essentially negligible.

The third observation is that at low Reynolds numbers, the airfoil's characteristics will be unsteady, with fluctuations in lift, drag, and pitching moment. This will make measuring the mean characteristics more challenging.

It is interesting to note that these observations indicate that it will be easier to obtain an accurate estimate of the mean characteristics of an airfoil by computational methods than experimentally.

\section{Acknowledgement}

The authors would like to acknowledge the generous support of this work by the Natural Sciences and Engineering Research Council of Canada.

\section{References}

[1] Selig, M.S., Donovan, J.F., and Fraser, D.B., Airfoils at Low Speeds, Soartech 8, Soartech Publications, Virginia Beach, U.S.A, 1989.

[2] Selig, M.S., Guglielmo, J.J., Broeren, A.P. and Giguere, P., Summary of Low Speeds Airfoil Data, Volume 1, Soartech Publications, Virginia Beach, U.S.A., 1995.

[3] Selig, M.S., Lyon, C.A., Giguere, P., Ninham, C.P. and Guglielmo, J.J., Summary of Low Speeds Airfoil Data, Volume 2, Soartech Publications, Virginia Beach, U.S.A., 1996.

[4] Lyon, C.A., Broeren, A.P, Giguere, P., Gopalarathnam, A. and Selig, M.S., Summary of Low Speeds Airfoil Data, Volume 3, Soartech Publications, Virginia Beach, U.S.A., 1997.

[5] R.F. Huang, and C.L. Lin, Vortex Shedding and Shear-Layer Instabilities of Wing at Low-Reynolds Numbers, AIAA Journal, 33(8), pp. 1398-1403, 1995.

[6] J.C.M. Lin and L.L. Pauley, Low-Reynold-Number Separation on an Airfoil, AIAA Journal, 34(8), pp. 1570-1577, 1996

[7] M. Raffel, C.E. Willert, J. Kompenhaus, Particle Image Velocimetry: A Practical Guide, Springer, 1998.

[8] H.W. Coleman and W.G. Steele, Engineering Application of Experimental Uncertainty Analysis, AIAA Journal, 33, pp. 1888-1896, 1995.

[9] A.K. Prasad, Particle Image Velocimetry, Current Science 79(1), pp. 51-60, 2000.

[10] D.J. Forliti, P.J. Strykowski and K. Debatin, Bias and Precision Errors of Digital Particle Image Velocimetry, Experiments in Fluids, 28(436), pp. 436-447, 2000. 